Kazuhiro Akaba • Toshiyuki Kimura • Ayako Sasaki

Saori Tanabe · Takashi Wakabayashi • Masahiko Hiroi

Seiji Yasumura • Kazuko Maki • Shogo Aikawa

Kiyoshi Hayasaka

\title{
Neonatal hyperbilirubinemia and a common mutation of the bilirubin uridine diphosphate-glucuronosyltransferase gene in Japanese
}

Received: June 16, 1998 / Accepted: August 5, 1998

\begin{abstract}
Neonatal hyperbilirubinemia, which is prevalent among Asian peoples, has been considered as a physiological phenomenon, and its metabolic basis has not been clearly explained. Gilbert syndrome is a common inherited disease of unconjugated hyperbilirubinemia due to decreased bilirubin uridine diphosphate-glucuronosyltransferase (BUGT), and its role in neonatal jaundice has recently been considered. We have previously reported that the Gly71Arg mutation of the $B-U G T$ gene associated with Gilbert syndrome is prevalent in Japanese, Korean, and Chinese populations and was more frequently detected in neonates with severe hyperbilirubinemia than in control subjects. We have studied 159 Japanese full-term neonates, evaluating the relationship between the B-UGT genotype and the severity of jaundice, as assessed with a transcutaneous bilirubinometer. The gene frequency of the Gly71Arg mutation in these neonates was 0.19 , and neonates carrying the Gly $71 \mathrm{Arg}$ mutation had significantly increased bilirubin levels on days 2-4, manifested in a gene dose-dependent manner. The frequency of the Gly71Arg mutation was 0.47 in the neonates who required phototherapy (i.e., those with more severe hyperbilirubinemia), significantly higher than 0.16 in the neonates who did not require the therapy. The gene frequency of the TA repeat promoter polymorphism, the $(\mathrm{TA})_{7}$ mutation, was 0.07 , and neonates carrying this mutation did not have an increase in bilirubin. These results
\end{abstract}

K. Akaba $\cdot$ T. Kimura - A. Sasaki $\cdot$ S. Tanabe $\cdot$ T. Wakabayashi K. Hayasaka $(\square)$

Department of Pediatrics, Yamagata University School of Medicine, Yamagata 990-2331, Japan

Tel. +81-23-628-5329; Fax +81-23-628-5332

e-mail:hayasaka@med.id.yamagata-u.ac.jp

M. Hiroi

Department of Obstetrics and Gynecology, Yamagata University

School of Medicine, Yamagata, Japan

S. Yasumura

Department of Public Health, Yamagata University School of

Medicine, Yamagata, Japan

K. Maki · S. Aikawa

Yamagata Prefectural Institute of Public Health, Yamagata, Japan suggested that the Gly71Arg mutation contributes to the high incidence of neonatal hyperbilirubinemia in Japanese.

Key words Bilirubin uridine diphosphate-glucuronosyltransferase $\cdot$ Neonatal hyperbilirubinemia - Gilbert syndrome

\section{Introduction}

Hyperbilirubinemia in newborn infants has been recognized as a common physiological phenomenon, based on the following observations: cessation of bilirubin clearance by placental transport; increased production of bilirubin probably due to the short lifespan of circulating erythrocytes, increased heme degradation of the fetal hematopoietic tissues, and increased turnover of cytochromes; low uptake of bilirubin in liver; low activity of hepatic bilirubin uridine diphosphate-glucuronosyltransferase (B-UGT); and persistent enterohepatic circulation of bilirubin (Yao and Stevenson 1995; Halamek and Stevenson 1997). It is also well known that there are significant differences in the severity of physiological jaundice among different ethnic populations. Severe neonatal hyperbilirubinemia is prevalent in Japanese, Korean, Chinese, and Native American newborns (Halamek and Stevenson 1997). Gilbert syndrome is a common inherited disease of unconjugated hyperbilirubinemia due to decreased B-UGT (Chowdhury et al. 1995). Molecular analysis has revealed that Gilbert syndrome was associated with the following mutations of the B-UGT gene: Gly71Arg, Pro229Gln, Arg367Gly, Tyr486Asp, and (TA) ${ }_{7}$ (Bosma et al. 1995; Aono et al. 1995; Koiwai et al. 1995; Monaghan et al. 1996). Kaplan et al. (1997) reported that a polymorphic two-base-pair addition (TA) in the promoter of the $B-U G T$ gene, the (TA $)_{7}$ mutation, increased the risk of neonatal hyperbilirubinemia in glucose-6-phosphate dehydrogenase deficiency. Recently, Bancroft et al. (1998) studied 151 healthy infants, and reported that newborns homozygous for the (TA $)_{7}$ mutation had an accelerated increase in neonatal jaundice during the 
first 2 days of life. We have reported that the Gly71Arg mutation of the $B-U G T$ gene is prevalent in Japanese, Koreans, and Chinese and was more frequently detected in Japanese newborns treated with phototherapy for hyperbilirubinemia (i.e., those with more severe hyperbilirubinemia) than in control subjects (Akaba et al. 1998).

Here we report on our investigations into whether the Gly71Arg mutation contributes to the high incidence of neonatal hyperbilirubinemia in Japanese.

\section{Subjects and methods}

\section{Subjects and DNA isolation}

The subjects $(n=159)$ were recruited from among the Japanese full-term neonates born at Yamagata University Hospital in 1997. The main characteristics of the 159 neonates were: male-to-female ratio, 81:78; gestational age $276 \pm 3$ (mean \pm SD) days; birth weight, $3068 \pm 401$ (mean \pm SD) g; and percentage breast fed, $22 \%$. Newborns with severe hyperbilirubinemia were treated with phototherapy according to the criteria we have described previously (Akaba et al. 1998). The number of observations was not constant during the study, because the data for infants undergoing phototherapy were eliminated after the initiation of therapy, and day 5 data were not available for four subjects homozygous for Gly71 (wild type). After informed consent was obtained from the parents, genomic DNA was isolated from spare dried blood spots from newborn screening cards, as previously described (Jinks et al. 1989). This study was approved by the Ethics Committee of Yamagata University School of Medicine.

Polymerase chain reaction (PCR) amplification and analysis of the B-UGT gene

Fragments encompassing the $5^{\prime}$ promoter region, including the TATAA element and exon 1A, were amplified by nested PCR based on the genomic information (Ritter et al. 1991; Ritter et al. 1992). We used the following PCR primers in this study: first PCR for promoter, PF: 5'GAGGTTCTGGAAGTACTTTG-3' and PR: 5'-TCCGTCTCTGATGTACAACG-3'; second PCR for promoter, PFIN: 5'-ACTTTGCTGTGTTCACTCAA-3' and PRIN: 5'-CAGCAGGCCCAGGACAAGTG-3'; first PCR for exon 1, 1F: 5'-AAGTAG GAGAGGGCGAACC-3' and 1R: 5'-GTCCCACTCCAATACACAC-3'; second PCR for exon 1, 1FIN: 5'-TGAAATAGTTGTCCTAGCACC-3' and 1RIN: $5^{\prime}$-CCATGAGCTCCTTGTTGTGC-3'. The size of the TA insertion was determined by sequencing. Primer PFIN was biotinylated at the $5^{\prime}$ end, and singlestrand DNA was prepared for sequence determination, using streptavidin-coated magnetic beads (Dynabeads M-280 Streptavidin; Dynal, Oslo, Norway). Sequences were deter- mined by the dideoxy chain termination method, using the primer SEQPRO: 5'-CAGCAGGCCCAGGACAAGTG$3^{\prime}$. For the detection of the Gly71Arg mutation, aliquots of the second PCR products of exon 1 were blotted on a Nylon membrane and allele-specific oligonucleotide hybridization (ASO) analysis was performed as previously described (Akaba et al. 1998).

\section{Hyperbilirubinemia assessment}

Neonatal hyperbilirubinemia was assessed with a Jaundice Meter (model 102; Minolta, Osaka, Japan) once a day, at the same time, during the first week of life (Yamanouchi et al. 1980). Assessment was expressed by the reading of the transcutaneous bilirubinometer (TCBR).

\section{Statistical analysis}

The gestational age and birth weight of three different genotypic groups we identified (see below) were compared using analysis of variance. The TCBR of the three groups was initially analyzed with the Kruskal-Wallis test. If significant differences were detected, comparison between each of the groups was performed with the Mann-Whitney $U$ test. Other analyses were performed by $\chi^{2}$ analysis.

\section{Results}

Distribution of the genotype of the $B-U G T$ gene in the 159 newborns is shown in Table 1 . The gene frequency of the Gly71Arg mutation in this study was 0.19 , which was not significantly different from 0.13 in our previous study (Akaba et al. 1998). Of the 159 newborns, 104 were homozygous for Gly71 (wild type), 48 were heterozygous for Arg71, and 7 were homozygous for Arg71. There was no significant difference in sex distribution, gestational age, birth weight, or type of feeding among these three groups (Table 2). As shown in Fig. 1, there were significant differences in TCBR on days 2-4 among the three groups. Homozygotes of Arg71 showed the highest TCBR among the three groups and heterozygotes of Arg71 had higher TCBR than homozygotes of Gly71. The gene frequency of the Gly71Arg mutation was 0.47 in the neonates who required

Table 1 Distribution of three genotypes of the bilirubin uridine diphosphate-glucuronosyltransferase (B-UGT) gene in 159 newborns

TATAA elements

$\begin{array}{lll}(\mathrm{TA})_{6} /(\mathrm{TA})_{6} & (\mathrm{TA})_{6} /(\mathrm{TA})_{7} & (\mathrm{TA})_{7} /(\mathrm{TA})_{7}\end{array}$

\begin{tabular}{lrrr}
\hline Amino acid residue at 71 & & & \\
Gly71/Gly71 & 89 & 13 & 2 \\
Gly71/Arg71 & 44 & 4 & 0 \\
Arg71/Arg71 & 7 & 0 & 0 \\
Total & 140 & 17 & 2 \\
\hline
\end{tabular}


Table 2 Subjects grouped by genotype of amino acid residue at 71

\begin{tabular}{|c|c|c|c|c|c|}
\hline & \multicolumn{3}{|c|}{ Amino acid residue at 71} & \multirow{2}{*}{\multicolumn{2}{|c|}{$P$ value }} \\
\hline & Gly71/Gly71 & Gly71/Arg71 & Arg71/Arg71 & & \\
\hline Number of subjects & 104 & 48 & 7 & & \\
\hline Male/Female & $55 / 49$ & $25 / 23$ & $1 / 6$ & $\left.0.14^{*}\right]$ & \\
\hline Gestational age (days) & $276 \pm 8$ & $275 \pm 8$ & $280 \pm 7$ & $0.22 * *$ & All \\
\hline Birth weight $(\mathrm{g})$ & $3093 \pm 405$ & $3038 \pm 383$ & $2898 \pm 464$ & $0.39 * *$ & NS \\
\hline Breast fed ${ }^{a}$ & $21(20)$ & $13(27)$ & $1(14)$ & $0.56 *$ & \\
\hline
\end{tabular}

Data values for gestational age and birth weight are expressed as means $\pm \mathrm{SD}$

$* \chi^{2}$ analysis; ** Analysis of variance. Numbers in parentheses represent percentages

NS, Not significant

${ }^{a}$ Breast led newborns

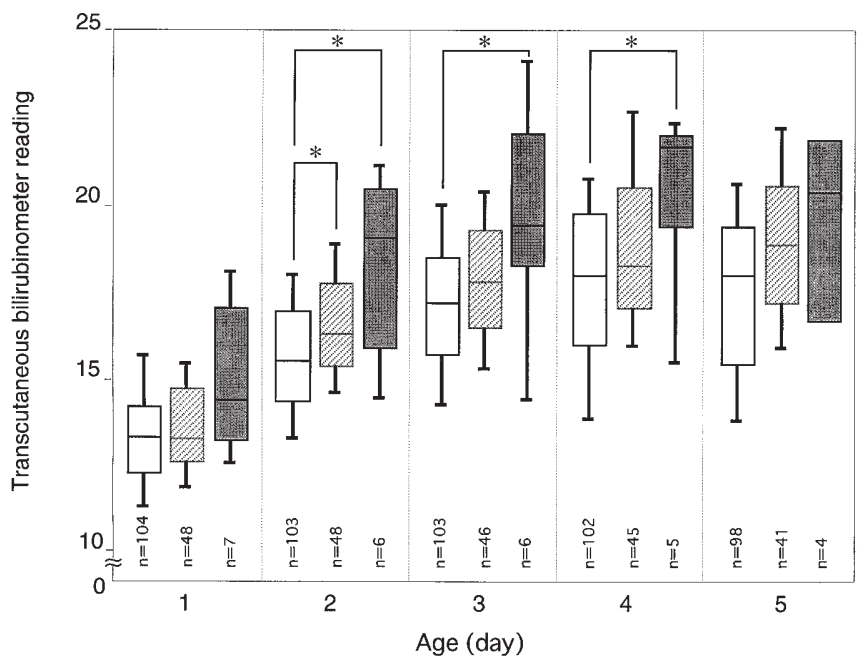

Fig. 1 Transcutaneous bilirubinometer reading from days 1 to 5 in the three genotype groups. (shown in Table 1) $\boxminus$ indicates the Gly71/Gly71 (wild type) genotype group. Hatched boxes indicates the Gly71/Arg71 (heterozygote) genotype group. Shaded boxes indicates the Arg71/ Arg71 (homozygote) genotype group. Boxes show median, 25\%, and $75 \%$, and bars are $10 \%$ and $90 \%$ for each group. Significant differences $(P<0.05)$ were seen on days 2,3 , and 4 with the Kruskal-Wallis test. *Significant differences $(P<0.05)$ between each group with the MannWhitney $U$-test. "n" represents numbers tested

the phototherapy, significantly higher than 0.16 in the neonates who did not require the therapy (Table 3 ).

As for the TA repeat promoter polymorphism, the gene frequency of $(\mathrm{TA})_{7}$ in this study was 0.07 , similar to 0.1 in our previous study (Akaba et al. 1998). Of the 159 newborns, 140 were homozygous for (TA) 6 (wild type), 17 were heterozygous for (TA) $)_{7}$, and 2 were homozygous for (TA) 7 . There was no significant difference in TCBR among these three groups (Kruskal-Wallis test, $P<0.22$ ). To exclude the effect of the Gly71Arg mutation, TCBR was compared among the subjects homozygous for Gly71 (wild type). However, only two babies were homozygous for (TA $)_{7}$ and Gly71; the median values of their TCBR on days $1-5$ were lower than those of homozygotes of (TA) 6 and Gly71, but the difference was not significant (Kruskal-Wallis test, $P<0.07)$.

These results suggest that the Gly71Arg mutation contributes to the high incidence of neonatal hyperbilirubinemia in Japanese.
Table 3 Phototherapy and Gly71Arg mutation

\begin{tabular}{|c|c|c|c|c|}
\hline & \multicolumn{3}{|c|}{ Amino acid residue at 71} & \multirow[b]{2}{*}{$\begin{array}{l}\text { Gly71Arg gene } \\
\text { frequency }\end{array}$} \\
\hline & $\begin{array}{l}\text { Gly71/ } \\
\text { Gly71 }\end{array}$ & $\begin{array}{l}\text { Gly71/ } \\
\text { Arg71 }\end{array}$ & $\begin{array}{l}\text { Arg71/ } \\
\text { Arg71 }\end{array}$ & \\
\hline PT & 5 & 8 & 4 & $0.47 *$ \\
\hline NonPT & 99 & 40 & 3 & $0.16^{*}$ \\
\hline Total & 104 & 48 & 7 & \\
\hline
\end{tabular}

* Significant differences $(P<0.001)$ seen by $\chi^{2}$ analysis.

PT, Subjects who received phototherapy; nonPT, subjects who did not require phototherapy.

\section{Discussion}

Neonatal jaundice has been considered a physiological phenomenon and its metabolic basis has not been clear. Neonatal hyperbilirubinemia is prevalent among Asian peoples, and increased bilirubin production in Japanese newborns was speculated in a study that measured blood carboxyhemoglobin levels (Fischer et al. 1988). Based on the study of the relationship between glucose-6-phosphate dehydrogenase deficiency and neonatal hyperbilirubinemia, it was suggested that the deficiency of B-UGT activity was the central rate-limiting step in the development of neonatal hyperbilirubinemia (Kaplan et al. 1997). Gilbert syndrome is a common and benign cause of constitutional jaundice that is due to decreased activity of hepatic B-UGT (Chowdhury et al. 1995). Molecular analysis has revealed that Gilbert syndrome was associated with several mutations of the B-UGT gene (Bosma et al. 1995; Aono et al. 1995; Koiwai et al. 1995; Monaghan et al. 1996). We have previously reported that the gene frequency of the Gly71Arg mutation associated with Gilbert syndrome was 0.13 in Japanese and 0.23 in Koreans and Chinese, and that the mutation was not detected in Germans (Akaba et al. 1998). It was also more frequently detected in Japanese newborns treated with phototherapy for hyperbilirubinemia than in control subjects. In this study we demonstrated that neonates carrying the Gly71Arg mutation had a significant increase in serum bilirubin level on days 2-4 compared with neonates not carrying the mutation, and manifested in a gene dose-dependent manner. The number of babies receiving phototherapy increased in the groups carrying the mutation until day 5 and their data were 
eliminated, and this may explain why there was no significant difference on day 5 .

Bancroft et al. (1998) reported that homozygotes of the $(\mathrm{TA})_{7}$ mutation had a greater increase in TCBR during the first 2 days of life than heterozygotes or homozygotes of the $(\mathrm{TA})_{6}$. The gene frequency of the $(\mathrm{TA})_{7}$ mutation is about 0.1 in Japanese, significantly lower than 0.4 in European peoples. (TA) ${ }_{7}$ and Gly71Arg mutations are allelic, and a difference in two gene frequencies among various populations is probably due to the so-called founder effect. We found only a few neonates carrying the (TA $)_{7}$ mutation, and our data showed that the (TA $)_{7}$ mutation did not increase TCBR, even among the homozygotes of Gly71 (wild type). It is clear that the Gly71Arg mutation contributes to the higher incidence of neonatal hyperbilirubinemia among Japanese. However, one-third of the neonates receiving phototherapy did not carry the Gly71Arg mutation and not all neonates carrying the Gly71Arg mutation, including homozygotes, were similarly affected with severe neonatal hyperbilirubinemia. It is probable that other predisposing factors may be associated with the neonatal hyperbilirubinemia in Japanese, and may override the effect of the (TA $)_{7}$ mutation.

Neonatal hyperbilirubinemia is a physiological phenomenon, and severe cases have been treated by phototherapy or exchange transfusion. However, it is important clinically to identify predisposing factors to establish a definitive treatment. Further studies are needed to clarify the mechanism of neonatal hyperbilirubinemia.

Acknowledgments We would like to thank Dr. John Christodoulou (Sydney University, Australia) for his critical reading and helpful suggestions, and Dr. H. Sato (Shiga University of Medical Science) for his kind advice in regard to our study. This work was supported in part by Grants from the Ministry of Health and Welfare of Japan.

\section{References}

Akaba K, Kimura T, Sasaki A, Tanabe S, Ikegami T, Hashimoto M, Umeda H, Yoshida H, Umetsu K, Chiba H, Yuasa I, Hayasaka K (1998) Neonatal hyperbilirubinemia and mutation of the bilirubin uridine diphosphate-glucuronosyltransferase gene: A common mutation among Japanese, Koreans and Chinese. Biochem Mol Biol Int 46: $21-26$

Aono S, Adachi Y, Uyama E, Yamada Y, Keino H, Nanno T, Koiwai O, Sato H (1995) Analysis of genes for bilirubin UDPglucuronosyltransferase in Gilbert's syndrome. Lancet 345: 958-959

Bancroft JD, Kreamer B, Gourley GR (1998) Gilbert syndrome accelerates development of neonatal jaundice. J Pediatr 132: 656-660

Bosma PJ, Chowdhury JR, Bakker C, Gantla S, de Boer A, Oostra BA, Lindhout D, Tytgat GN, Jansen PL, Oude Elferink RPJ, Chowdhury NR (1995) The genetic basis of the reduced expression of bilirubin UDP-glucuronosyltransferase 1 in Gilbert's syndrome. N Engl J Med 333: 1171-1175

Chowdhury JR, Wolkoff AW, Chowdhury NR, Arias IM (1995) Hereditary jaundice and disorders of the bilirubin metabolism. In: Scriver CR, Beaudet AL, Sly WS, Valle D (eds) The metabolic and molecular bases of inherited disease. Mc-Graw-Hill, New York, pp 2161-2208

Fischer AF, Nakamura H, Uetani Y, Vreman HJ, Stevenson DK (1988) Comparison of bilirubin production in Japanese and Caucasian infants. J Pediatr Gastroenterol Nutr 7: 27-29

Halamek LP, Stevenson DK (1997) Neonatal jaundice and liver disease. In: Fanaroff AA, Martin RJ (eds) Neonatal-perinatal medicine: Diseases of the fetus and infant. Mosby, St. Louis, pp 1345-1389

Jinks DC, Minter M, Tarver DA, Vanderford M, Hejtmancik JF, McCabe ER (1989) Molecular genetic diagnosis of sickle cell disease using dried specimens on blottters used for newborn screening. Hum Genet 81: 363-366

Kaplan M, Renbaum P, Levy-Lahad E, Hammerman C, Lahad A, Beutler E (1997) Gilbert syndrome and glucose-6-phosphate dehydrogenase deficiency: A dose-dependent genetic interaction crucial to neonatal hyperbilirubinemia. Proc Natl Acad Sci USA 94: 12128 12132

Koiwai O, Nishizawa M, Hasada K, Aono S, Adachi Y, Mamiya N, Sato H (1995) Gilbert's syndrome is caused by a heterozygous missense mutation in the gene for bilirubin UDPglucuronosyltransferase. Hum Mol Genet 4: 1183-1186

Monaghan G, Ryan M, Seddon R, Hume R, Burchell B (1996) Genetic variation in bilirubin UDP-glucuronosyltransferase gene promoter and Gilbert's syndrome. Lancet 347: 578-581

Ritter JK, Crawford JM, Owens IS (1991) Cloning of human liver bilirubin UDP-glucuronosyltransferase cDNAs with expressin in COS-1 cells. J Biol Chem 266: 1043-1047

Ritter JK, Chen F, Sheen YY, Tran HM, Kimura S, Yeatman MT, Owens IS (1992) A novel complex locus UGT1 encodes human bilirubin, phenol, and other UDP-glucuronosyltransferase isozymes with identical carboxyl termini. J Biol Chem 267: 3257-3261

Yamanouchi I, Yamauchi Y, Igarashi I (1980) Transcutaneous bilirubinometry: Preliminary studies of noninvasive transcutaneous bilirubin meter in the Okayama National Hospital. Pediatr 65: 195202

Yao TC, Stevenson DK (1995) Advances in the diagnosis and treatment of neonatal hyperbilirubinemia. Clin Perinatol 22: 741-758 